\title{
A Survey on Comparison of ABC method with other Optimization methods by using PEGASIS Protocol
}

\author{
Chandan Verma ${ }^{1}$, Ashish Sharma ${ }^{2}$ \\ M.Tech. Student, Department of Computer Engineering, BIMT Shimla, India ${ }^{1}$ \\ Assistant Professor, Department of Computer Engineering, BIMT Shimla, India ${ }^{2}$
}

\begin{abstract}
Routing is the major challenge for sensor networks. It presents the trade- off between efficiency as well as responsiveness. There are various protocols existing in this category. In this work, we analyse Power Efficient Gathering in Sensor Information System (PEGASIS) hierarchical protocol for routing with the protocols on the basis of total energy consumed, sensors lifetime and provides a comparison with traditional methods. The optimization method used in this proposed work is ABC (Artificial Bee Colony) optimization method. Utilization of PEGASS is done because PEGASIS is a redirecting method, so allows good routing. This method employs some sort of greedy approach beginning from the actual furthermost node and each of the sensor nodes form some sort of string just like composition. Performance of whole simulation is being done using various metrics like Number of alive nodes, Data Packets received at base station and Energy consumption.
\end{abstract}

Keywords: WSN, PEGASIS, ABC, Sensor nodes.

\section{INTRODUCTION}

A Wireless Sensor Network (WSN) consists of a set of nodes of typically low performance. They collaborate with each other to perform sensing tasks in a given environment. A wireless sensor network may contain one or more sink nodes (Base Stations) to collect sensed data and relay it to a central processing and storage system. A sensor node is typically powered by a battery and can be divided into three main functioned units, which are: a sensing unit, a communication unit and a processor unit. Recent advances in micro-electro-mechanical systems technology, wireless communications and digital electronics have boosted the development of sensor nodes. This brings the blooming prospect of WSNs into practical feasibility [1].

Despite the innumerable applications of WSNs, these networks have several restrictions, e.g., limited energy supply, limited computing power, and limited bandwidth of the wireless links connecting sensor nodes. One of the main design goals of WSNs is to carry out data communication while trying to prolong the lifetime of the network and prevent connectivity degradation by employing aggressive energy management techniques. The design of routing protocols in WSNs is influenced by many challenging factors. These factors must be overcome before efficient communication that can be achieved in WSNs. In the following, we summarize some of the routing challenges and design issues that affect routing process in WSN [2].

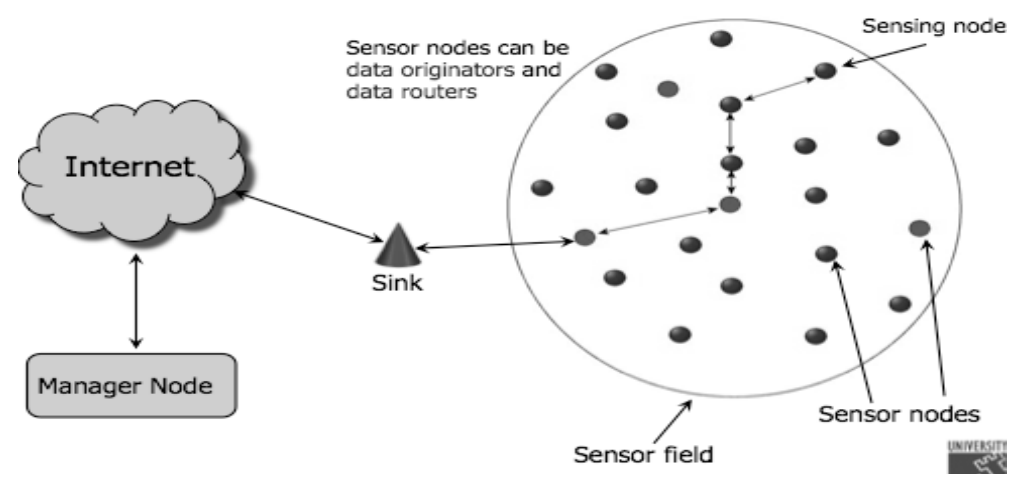

Fig. 1 Sensor Networks

Node deployment: Node deployment in WSNs is application dependent and affects the performance of the routing protocol. The deployment can be either deterministic or randomized. In deterministic deployment, the sensors are 
manually placed and data is routed through pre-determined paths. However, in random node deployment, the sensor nodes are scattered randomly creating an infrastructure in an ad-hoc manner. If the resultant distribution of nodes is not uniform, optimal clustering becomes necessary to allow connectivity and enable energy efficient network operation. Inter-sensor communication is normally within short transmission ranges due to energy and bandwidth limitations. Therefore, it is most likely that a route will consist of multiple wireless hops [3].

Energy consumption without losing accuracy: Sensor nodes can use up their limited supply of energy performing computations and transmitting information in a wireless environment. As such, energy conserving forms of communication and computation are essential. Sensor node lifetime shows a strong dependence on the battery lifetime. In a multi-hop WSN, each node plays a dual role as data sender and data router. The malfunctioning of some sensor nodes due to power failure can cause significant topological changes and might require re-routing of packets and reorganization of the network.

Data Reporting Model: Data sensing and reporting in WSNs is dependent on the application and the time criticality of the data reporting. Data reporting can be categorized as time-driven (continuous), event-driven, query-driven and hybrid. The time-driven delivery model is suitable for applications that require periodic data monitoring. As such, sensor nodes will periodically switch on their sensors and transmitters, sense the environment and transmit the data of interest at constant periodic time intervals. In event-driven and query-driven models, sensor nodes react immediately to sudden and drastic changes in the value of a sensed attribute due to the occurrence of a certain event or a query is generated by the BS. As such, these are well suited for time critical applications. A combination of the previous models is also possible. The routing protocol is highly influenced by the data reporting model with regard to energy consumption and route stability [4].

Node/Link Heterogeneity: In many studies, all sensor nodes were assumed to be homogeneous, i.e., having equal capacity in terms of computation, communication, and power. However, depending on the application a sensor node can have different role or capability. The existence of heterogeneous set of sensors raises many technical issues related to data routing. For example, some applications might require a diverse mixture of sensors for monitoring temperature, pressure and humidity of the surrounding environment, detecting motion via acoustic signatures, and capturing the image or video tracking of moving objects. These special sensors can be either deployed independently or the different functionalities can be included in the same sensor nodes. Even data reading and reporting can be generated from these sensors at different rates, subject to diverse quality of service constraints, and can follow multiple data reporting models. For example, hierarchical protocols designate a cluster head node different from the normal sensors. These cluster heads can be chosen from the deployed sensors or can be more powerful than other sensor nodes in terms of energy, bandwidth, and memory. Hence, the burden of transmission to the BS is handled by the set of cluster-heads [5].

\section{PEGASIS PROTOCOL}

PEGASIS is a near optimal chain-based routing protocol that is an improvement over LEACH. The main idea in PEGASIS protocol is for node to receive from and transmit to close neighbours and take turns for being the leader for transmission of data to BS. This approach distributes the energy load evenly among the sensor nodes. The nodes randomly placed in the field, organize themselves in the form of chain using greedy algorithm. Alternatively, BS computes this chain and broadcasts it to all the nodes. Fig 2 shows node 0 and node 1 connecting to node 3 , node 3 connecting to node 1 and node 1 connecting to node 2 . When a node dies, the chain is constructed in the same manner to bypass the dead node [6].

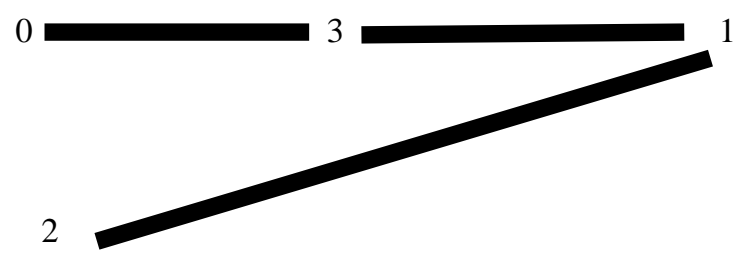

Fig. 2 Chain Formation in PEGASIS

\section{ARTIFICIAL BEE COLONY (ABC)}

In the $\mathrm{ABC}$ model, the colony consists of three groups of bees: employed bees, onlookers and scouts. It is assumed that there is only one artificial employed bee for each food source. In other words, the number of employed bees in the 
colony is equal to the number of food sources around the hive. Employed bees go to their food source and come back to hive and dance on this area [7]. The employed bee whose food source has been abandoned becomes a scout and start to search for finding a new food source. Onlookers watch the dances of employed bees and choose food sources depending on dances.

The main steps of the algorithm are given below:

- Initialize population.

- Evaluate population.

- For cycle 1.

- Repeat.

- Find food source positions

- Apply greedy selection to get new solutions.

- Calculate the probability values for all solutions.

- Get new positions of food.

- Again apply greedy process.

- Optimize the position of food to get new positions.

- Do for cycle + cycle+1

- Until cycle = MCN.

\section{OBJECTIVES}

The objectives are declared below:

1. To implement Wireless Sensor Network by deploying number of nodes in the designed network.

2. Utilize ABC Algorithm within each sensor node to develop the network performance efficiently in terms of broadcasting the messages so that node will consume less energy using PEGASIS protocol.

3. Finding out the best possible values of the fitness function.

4. Compare ABC method with other optimization methods.

5. Measure the performance of the network based on performance metrics.

\section{LITERATURE SURVEY}

- Sandra Sendra et.al 2011[8] proposed that wireless sensor systems have turn out to be progressively famous because of their extensive variety of uses. Energy utilization is one of the greatest requirements of the wireless sensor hub and this constraint consolidated with a common arrangement of substantial number of hubs that are adding numerous difficulties to the configuration and administration of wireless sensor systems. They are regularly utilized for wireless environment observing as a part of regions where giving electrical energy is troublesome. Thusly, the gadgets should be fuelled by batteries and option energy sources. Since battery energy is constrained, the utilization of distinctive methods for energy conserving is one of the most smoking subjects in WSNs. In this work, they show a study of energy saving as well as also energy optimization systems for wireless sensor networks, which improves the ones in presence and acquaints the pursuer with the most surely understood accessible routines that can be utilized to conserve energy. They are investigated from a few perspectives: Device hardware, transmission, MAC and routing protocols.

- Raghavendra V. Kulkarni et.al 2010 [9] presented that wireless sensor systems (WSNs) are the systems of selfgoverning hubs utilized for observing a domain. Engineers of WSNs face challenges that emerge from communication link catastrophes, memory and computational requirements, and constrained energy. Numerous issues in WSNs are defined as multidimensional advancement issues, and drew nearer through bio-propelled strategies. Particle swarm optimization is a basic, successful and computationally proficient improvement algorithm. It has been connected to address WSN issues, for example, optimal deployment, hub localization, clustering in addition to information-accumulation. This paper talks about WSNs, which presents PSO and examines its suitability for WSN applications. It additionally exhibits a brief review of how PSO is customized to address these issues.

- Gogu, A. et.al 2011 [10] presented that as WSNs turn out to be progressively pervasive, a great comprehension of these issues as far as hypothetical intricacy is of extraordinary help in planning suitable algorithms. In this paper, they analyze probably the most central optimization issues identified with coverage, topology control, scheduling, routing and mobility in WSNs. At that point they concentrate on their unpredictability and investigate the distinctions that exist with counterpart hypothetical issues or those effectively considered in conventional systems. They present as they'll some of the primary strategies proposed in the writing and report some open issues with respect to these issues. 
- Debmalya Bhattacharyalet.al 2011 [11] proposed that WSNs comprises of a system of wireless hubs that have the ability to sense a parameter of hobby. Sensors of different sorts are sent universally and pervasively in shifted situations, for example, office structures, untamed life stores, war zones, versatile systems, and so forth. The detected parameter is transferred to a base station through the system shaped amongst these hubs. The gadgets utilized are commonly portrayed by minimal effort, low power and are rough in operation. The hub incorporates programming, reckoning, correspondence, and detecting onto a solitary framework and gives a simple client interface to working and sending it. The paper exhibits such a configuration which minimizes cost and energy consumption, in this way upgrading the life time of the hub.

- Jun Luo et.al [12] concentrated on abusing versatility to enhance the system lifetime of a WSN. They show a general advancement structure that has the capacity catch a few parts of augmenting system lifetime (MNL) including portable substances. Taking into account this structure, they lead a top to bottom investigation on each of these viewpoints furthermore of these features and also refer to procedures which can be utilized to take care of the subsequent optimization issues. They additionally exhibit certain numerical results where engineering of knowledge can be gained.

- M. M. Chandane et.al 2013 [13] proposed a re-enactment model for WSN. Writing study is done on energy-aware routing procedures, in which, the aforementioned is found in which, Minimum Total Transmission Power Routing and Minimum Battery Cost Routing Protocol, most extensively catches exchange offs of energy proficiency in addition to and system lifetime individually. Proposed re-enactment model is actualized utilizing Qualnet 4.5 and connected to MTPR and MBCR to dissect its execution. Through this study, they establish a framework for further research take a shot at improvements in augmenting the system lifetime of WSN. Trial result demonstrates that there is dependably an exchange off between energy productivity and system lifetime.

- Y. Sankarasubramaniam et.al 2003 [14] shown the subject of optimal package intermediate sensors before coming to the sink. Not at all like past work on package length optimization in other words, wireless sensor networks, energy efficiency is picked as total information before handing-off it to its neighbour the is proposed hub. Their point in this work is to focus the optimal information in light of the restricted and administration in package size for correspondence between neighboring sensor systems.

- Yuebin Bai et.al, 2008 [15] recommend an Energy Optimization Methodology taking into account Cross-Layer for Wireless Sensor Networks named as EOA, which consider the joint ideal outline of the physical, medium access control (MAC), and routing layer. The centre of EOA is on the reckoning of ideal transmission power, routing, and obligation cycle plan that streamline the WSNs energy-proficiency. They first suggest a feedback procedure which also calculates best possible transmission power level amongst nodes. At that point, routing protocol could make utilization of the transmission control as a metric by picking course with optimal power consumption in the direction of forwarding packages. At last, the cross-layer routing data is misused to shape an obligation cycle plan in MAC layer. EOA is accepted on a CROSSBOW's MicaZ bit stage, and assessed utilizing the TOSSIM test system; the recreation results demonstrate that EOA is an energy-proficient methodology and ready to accomplish huge execution change too.

\section{PROBLEM STATEMENT}

Many routing, power management, and data dissemination protocols have been specifically designed for WSNs where routing is an essential design issue. The focus, however, has been given to the routing protocols which might differ depending on the application and network architecture. Routing in WSNs is very challenging due to the inherent characteristics that distinguish these networks from other wireless networks like mobile ad hoc networks or cellular networks. First, due to the relatively large number of sensor nodes, it is not possible to build a global addressing scheme for the deployment of a large number of sensor nodes as the overhead of ID maintenance is high. Thus, traditional IP-based protocols may not be applied to WSNs.

Furthermore, sensor nodes are deployed in an ad-hoc manner. So, in proposed work routing problem will be solved using $A B C$ routing protocol on PEGASIS protocol. The whole simulation is done in MATLAB 2010a environment using various parameters i.e.

- Number of alive nodes: This instantaneous measure reflects the total number of nodes and that of each type that has not yet expended all of their energy.

- Data Packets received at base station: It is total number of data packets or messages that are received by the base station. This is also a measure of amount of information sent to BS from the sensor field. This measure varies linearly for all protocols.

- Energy consumed: It measures the instantaneous amount of energy being consumed in the network per round. This is simply the energy difference from the beginning till the end of a round. 


\section{METHODOLOGY USED}

Step 1 : Start

Step 2 : Initialize No. of nodes

Step 3 : Initialize length

Step 4 : Initialize Width

Step 5 : Assume pre-defined energy

Step 6 : Get number of nodes in the box.

Step 7 : Take random energy value

Step 8 : Plot X and Y location

Step 9 : Calculate energy of each block

Step 10 : Define source node

Step 11 : Find failed Block

Step 12 : Checking whether the source block and the failed block are similar or not.

Step 13 : Find Destination Block.

Step 14 : Checking that the destination node is not equal to the source node and the failed node

Step 15 : if yes then des $=1$

Step 16 : This identifies that the source block and the failed block are not similar

Step 17 : Cluster Head Fails

Step 18 : Call ABC optimization function.

Step 19 : Find final route through which the data will travel

Step 20 : Check if the node is not similar

Step 21 : Applying the second condition where energy is also a constraint

Step 22 : Optimize Qos parameters using fitness function

Step 23 : Plot Qos parameters

Step 24 : Stop

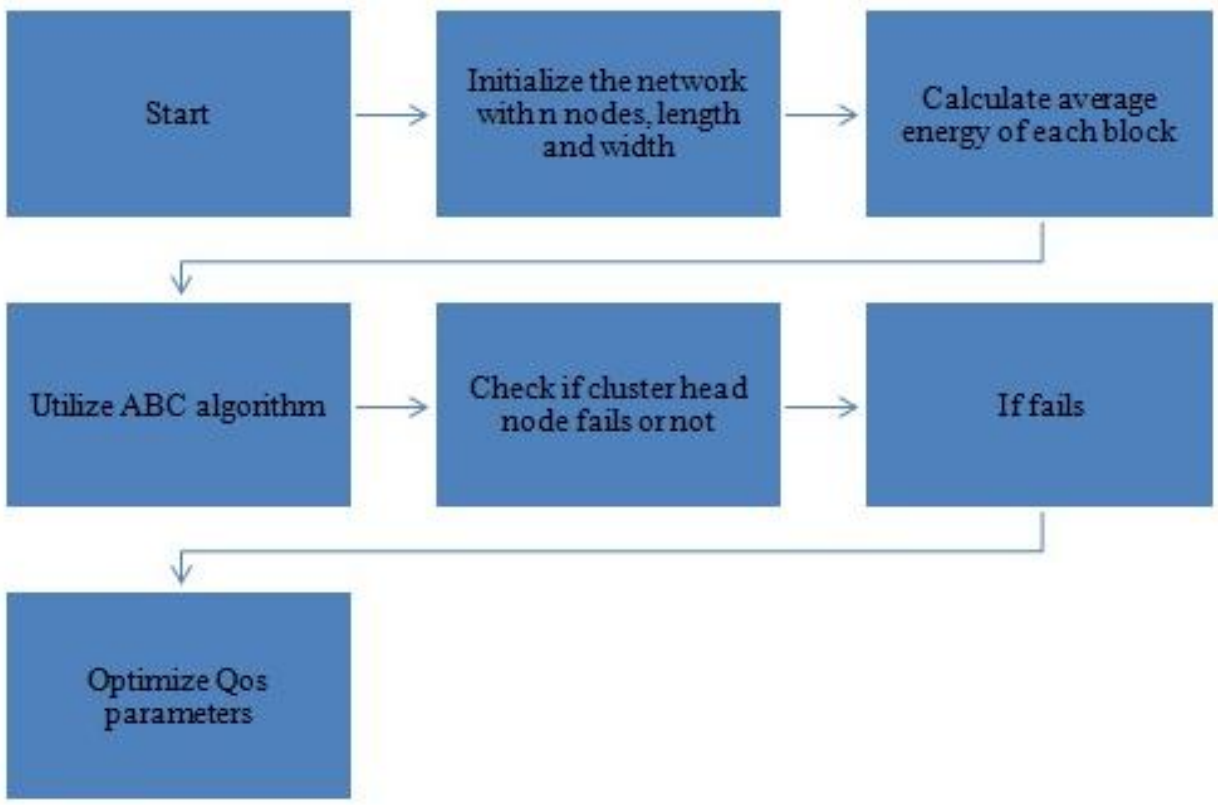

Fig.3 Proposed Flowchart

\section{CONCLUSION}

Step 25: Power-Efficient Gathering in Sensor Information Systems (PEGASIS), which is optimal for data gathering application in sensor network, will be used. The basic idea in PEGASIS is to execute a chain between the sensor nodes so that each node would receive from and transfers to a close neighbour. ABC (Artificial Bee Colony Algorithm) will be used in each sensor node for developing the efficiency of the network performance by means of broadcasting for less utilization of energy by using PEGASIS protocol. Comparison of $\mathrm{ABC}$ method with other optimization methods will takes place. The performance of the network will be measured by parameters, namely, Number of alive nodes, Data packets received at base stations and the consumption of energy. 


\section{REFERENCES}

[1] Elson and A. Parker. Tinker, “A tool for designing data-centric sensor networks", Information Processing in Sensor Networks, The Fifth International Conference, pp. 350-357, 2006.

[2] R. Ramadan, K. Abdelghany, and H. El-Rewini, "SensDep: a design tool for the deployment of heterogeneous sensing devices", In Second IEEE Work- shop on Dependability and Security in Sensor Networks and Systems, 2006.

[3] KP Ferentinos and TA Tsiligiridis, "Heuristic dynamic clustering in wire- less sensor networks towards uniform sensing", 15 th IST Mobile \& Wireless Communications Summit, Myconos, Greece, June, 2006.

[4] Eduardo F. Nakamura, Antonio A. F. Loureiro, and Alejandro C. Frery, "Information fusion for wireless sensor networks: Methods, models, and classifications", ACM Computing Survey, vol.39, 2007.

[5] Sandra Sendra, Jaime Lloret, Miguel García and José F. Toledo, "Power saving and energy optimization techniques for Wireless Sensor Networks", IEEE, vol.6 pp. 439-452, 2011.

[6] FengSen, Qi Bing and Tang Liangrui, “An Improved Energy-Efficient PEGASIS-Based Protocol in Wireless Sensor Networks", IEEEEighth International Conference on Fuzzy Systems and Knowledge Discovery, pp.2230-2233, 2011.

[7] S.Mohanty and S.K.Patra, "A novel Bio-inspired Clustering algorithm for Wireless Sensor Networks", 3rd International Conference on Intelligent and Advanced Systems, Kuala Lumpur, 2010.

[8] Sandra Sendra, Jaime Lloret, Miguel García and José F. Toledo, "Power saving and energy optimization techniques for Wireless Sensor Networks", IEEE, vol.6 pp. 439-452, 2011.

[9] Raghavendra V. Kulkarni and Ganesh Kumar Venayagamoorthy, "Particle Swarm Optimization in Wireless Sensor Networks: A Brief Survey", IEEE, 2010.

[10] Gogu, A, Nace, D and Dilo, A., “Optimization Problems in Wireless Sensor Networks”,IEEE, pp. 302-309 2011.

[11] Debmalya Bhattacharya1 and R.Krishnamoorthy, "Power Optimization in Wireless Sensor Networks", IJCSI, vol.8, pp.415-419, 2011.

[12] Jun Luo and Liu Xiang, "Prolong the Lifetime ofWireless Sensor Networks through Mobility: A General Optimization Framework", IEEE, pp. 583-590.

[13] M. M. Chandane, S. G. Bhirud and S. V. Bonde, "Routing Protocols in WSN, Mobile Communication and Power Engineering Communications in Computer and Information Science, vol. 296, pp 33-40, 2013.

[14] Y. Sankarasubramaniam, I. E Akyildiz and S. W. Mchughli, "Energy Efficiency based Packet Size Optimization in Wireless Sensor Networks", IEEE, 2003.

[15] Yuebin Bai, Shujuan Liu, Mo Sha2 Yang Lu and Cong Xu, "An Energy Optimization Protocol Based on Cross-Layer for Wireless Sensor Networks", IEEE, vol.3, pp.27-33, 2008. 